\title{
Maximizing the Number of Connections in Multifiber WDM Chain, Ring and Star Networks ${ }^{\star}$
}

\author{
Katerina Potika \\ Computer Science, ECE, National Technical University of Athens, Greece \\ epotik@cs.ntua.gr
}

\begin{abstract}
We study a wavelength assignment maximization problem in multifiber WDM networks. Given a network $G$, a set of requests, the number of fibers per link and the number of wavelengths, we want to maximize the number of requests that can be satisfied simultaneously. We propose polynomial time algorithms, that are either optimal or have a guaranteed worst case performance in basic topologies, such as chain, ring and star networks.
\end{abstract}

\section{Introduction}

A recent advance in WDM technology is the use of multiple fibers per link. In such networks signals that pass through the same link may use the same wavelength under the constraint that they traverse different parallel fibers.

We are given a network $G=(V, E)$, a function $\mu: E \rightarrow \mathbb{N}$, that defines the multiplicity of fibers on each link, a set of paths $\mathcal{P}$ (each request is prerouted) and $w$ colors. A path can be satisfied if a wavelength (color) is assigned along that path (coloring). A path multicoloring for $(G, \mathcal{P}, \mu, w)$ is valid, if for each edge $e$, paths from $\mathcal{P}$ that pass through $e$ are colored so that any color, out of the $w$, is used at most $\mu(e)$ times.

We study problem Maximum Path Multicoloring (Max-PMC). Given a graph $G=(V, E)$ with edge weights $\mu: E \rightarrow \mathbb{N}$, a set of paths $\mathcal{P}$ and a number $w$, find a valid path multicoloring of a subset $\mathcal{P}^{\prime} \subseteq \mathcal{P}$ with $w$ colors such that $\left|\mathcal{P}^{\prime}\right|$ is maximized.

A well known problem, that is a simplified version of our MAX-PMC, is the Maximum Integral Multicommodity Flow (MaX-MF). Given a graph $G=(V, E)$ with edge capacities $c: E \rightarrow \mathbb{N}$ and a set of flows $\mathcal{P}$ find a valid, with respect to $c$, path packing of $\mathcal{P}$, i.e., a subset $\mathcal{P}^{\prime} \subseteq \mathcal{P}$ such that no more than $c(e)$ paths in $\mathcal{P}^{\prime}$ go over edge e. The goal is to maximize $\left|\mathcal{P}^{\prime}\right|$.

Problem MAX-PMC in rings and stars is $\mathcal{N} \mathcal{P}$-hard because the single fiber problem MAX-PC is $\mathcal{N} \mathcal{P}$-hard in rings ([1]) and stars ([2]). For $\mathcal{N} \mathcal{P}$-hard prob-

\footnotetext{
* Research supported in part by the Greek Ministry of Education through grants "Pythagoras" and "Protagoras", of the EU funded program EPEAEK.
} 
lems we use approximation algorithms. An algorithm $A$ for a maximization problem $\Pi$ is a $\rho$-approximation (for $\rho>1$ ) if for every instance $I$ of $\Pi, A$ runs in time polynomial in $|I|$ and delivers a solution with cost $S O L \geq 1 / \rho \cdot O P T$, where $O P T$ denotes the cost of an optimal solution for $I$.

\section{Related Work}

For MAX-PMC in trees a 2.54-approximation algorithm is presented in 3 . Saad and Luo in [4] study problem MAX-PMC without assuming a special topology. They propose a well known method that considers only one wavelength at a time, and can be iteratively used for any number of wavelengths. In [5] they solve MAX-PMC using two heuristics in arbitrary topologies with uniform number of available fibers per link.

An interesting related problem for single-fiber networks is the MAXIMUM Path Coloring Problem (MaX-PC). In chains it can be solved exactly [ . Algorithms for MAX-PC in rings with 3/2-approximation is given in [1] and in stars with 1.58-approximation [2].

\section{Our results}

In chain networks we prove that MAX-PMC can be solved optimally. In ring networks we present for MAX-PMC a $1+\frac{1}{\mu_{\min }-2}$-approximation algorithm, where $\mu_{\min }$ is the minimum edge multiplicity that occurs in the ring network. Furthermore, we show that the existence of an exact algorithm for the MAX-MF for a network implies a $1 /\left(1-e^{-1}\right)$-approximation algorithm for MAX-PMC in this network. Applying this algorithm for ring and star networks we obtain two 1.58-approximation algorithms.

\section{Technical Preliminaries}

A multifiber network can be modeled by an undirected graph $G=(V, E)$. We use $n=|V|$ and $m=|\mathcal{P}|$. For a set $\mathcal{P}$ and an edge $e$ we denote by $L(e, \mathcal{P})$ the load of edge $e$, that is the number of paths in $\mathcal{P}$ that use $e$. The number of paths that can be colored in each edge is at most $\mu(e) \cdot w$. If we denote by $\mathcal{P}^{\prime}$ the subset of satisfied paths then the following condition must hold in each edge $e$ :

$$
L\left(e, \mathcal{P}^{\prime}\right) \leq \mu(e) \cdot w
$$

An upper bound of the optimal solution of an instance $I=(G, \mathcal{P}, \mu, w)$ for MAXPMC is the optimal solution of an instance $I^{\prime}=(G, \mathcal{P}, \mu \cdot w)$ for MAX-MF:

$$
O P T(I) \leq O P T_{\text {MAX-MF }}\left(I^{\prime}\right)
$$

We will also use as a subroutine an algorithm in chains for problem Path Multicoloring with Minimum Number of Collisions (MinColLisions-PMC). In this problem we are given a set of paths $\mathcal{P}$ and the number of wavelengths, and we want to satisfy all paths in $\mathcal{P}$ by finding for every edge an appropriate fiber multiplicity. Problem MinColLisions-PMC in chains can be solved optimal by using algorithm [7. 


\section{Chains}

For chains we propose Algorithm 1. We can solve problem MAX-MF in chains optimally using a algorithm described in [8] in $O(n+m)$ time.

Algorithm 1. MAX-PMC in chains

Input: chain $G=(V, E)$, a function $\mu: E \rightarrow \mathbb{N}$, a set of paths $\mathcal{P}$ and $w$ colors. Output: a maximum subset $\mathcal{P}^{\prime} \subseteq \mathcal{P}$ and a multicoloring of $\mathcal{P}^{\prime}$

1. Call algorithm for MAX-MF in chains for instance $(G, \mathcal{P}, \mu \cdot w)$, denote the returned solution by $\mathcal{P}^{\prime}$

2. Color instance $\left(G, \mathcal{P}^{\prime}, w\right)$ with algorithm for MinColLisions-PMC in chains

3. Return a multicoloring of the set of paths $\mathcal{P}^{\prime}$

Theorem 1. Algorithm 1 solves optimally problem MAX-PMC in chains.

Proof. We obtain a valid multicoloring of all paths in $\mathcal{P}^{\prime}$ using no more than $w$ colors. Algorithm for problem MinCollisions-PMC in chains returns an optimal solution, i.e. in each edge $e$, the number of paths in $\mathcal{P}^{\prime}$ that pass through $e$ and have the same color, is at most $\left\lceil\frac{L\left(e, \mathcal{P}^{\prime}\right)}{w}\right\rceil$. Using condition (11) we have that the number of color repetitions in each edge $e$ is $\left\lceil\frac{L\left(e, \mathcal{P}^{\prime}\right)}{w}\right\rceil \leq\left\lceil\frac{\mu(e) \cdot w}{w}\right\rceil \leq \mu(e)$, because $\mu(e)$ is an integer. Thus, the available multiplicities $\mu(e)$ suffice and in each edge we color $L\left(e, \mathcal{P}^{\prime}\right)$ paths.

\section{Rings}

The proposed algorithm is Algorithm 2, Algorithm for MAX-MF in rings 8 gives an optimal solution, denoted by $\mathcal{M}$.

Theorem 2. Algorithm 2 for MAX-PMC in rings is a $\frac{\mu_{\min }-1}{\mu_{m i n}-2}-$ approximation algorithm, where $\mu_{\min }=\min _{e \in E} \mu(e)$.

Proof. We first have to show that set $\mathcal{M}-\mathcal{P}_{0}$ can be valid multicolored. Algorithm transforms the ring in a chain by 'unfolding' the ring in node $v_{0}$. Doing that the load on some edges will be split in two parts, but on these edges we removed at most $w$ paths (see Figure1). Instead of one edge $e$ in the ring instance we may have two edges $e^{\prime}$ and $e^{\prime \prime}$ in the chain instance. In the edges $e$, in which the load is split, the number of times each color is repeated is at most:

$$
\begin{gathered}
\left\lceil\frac{L\left(e^{\prime}, \mathcal{M}\right)}{w}\right\rceil+\left\lceil\frac{L\left(e^{\prime \prime}, \mathcal{M}\right)-L\left(e, \mathcal{P}_{0}\right)}{w}\right\rceil \leq\left\lceil\frac{L\left(e^{\prime}, \mathcal{M}\right)}{w}\right\rceil+\left\lceil\frac{L\left(e^{\prime \prime}, \mathcal{M}\right)-w}{w}\right\rceil \leq \\
\left\lceil\frac{L\left(e^{\prime}, \mathcal{M}\right)+L\left(e^{\prime \prime}, \mathcal{M}\right)}{w}\right\rceil+1-1 \leq\left\lceil\frac{L(e, \mathcal{M})}{w}\right\rceil \leq\left\lceil\frac{\mu(e) w}{w}\right\rceil \leq \mu(e)
\end{gathered}
$$

The two last lines of the above equation holds because of condition (11) and because $\mu(e)$ is an integer. From equation (2) and from the facts that the solution 


\section{Algorithm 2. MAX-PMC in rings}

Input: ring $G=(V, E)$, a function $\mu: E \rightarrow \mathbb{N}$, a set of paths $\mathcal{P}$ and $w$ colors. Output: a maximum subset $\mathcal{P}^{\prime} \subseteq \mathcal{P}$ and a multicoloring of $\mathcal{P}^{\prime}$

1. Call algorithm for MAX-MF in rings for instance $(G, \mathcal{P}, \mu \cdot w)$, denote the returned solution, by $\mathcal{M}$.

2. Each edge $\left(v_{i}, v_{i+1}\right)$ is denoted by $e_{i}$. Find node $e_{0}$ with minimum multiplicity and reindex nodes accordingly.

3. Find the paths in $\mathcal{M}$ that pass through node $v_{0}$, denote them by $\mathcal{Q}$. Sort the paths in $\mathcal{Q}$ in descending order of the index of their clockwise endpoint and choose the $w$ first paths, denote them by $\mathcal{P}_{0}$.

4. Set $\mathcal{P}^{\prime}=\mathcal{M}-\mathcal{P}_{0}$.

5. Transform the ring $\left(G, \mathcal{P}^{\prime}\right)$ to a chain $\left(G^{\prime}, \mathcal{P}^{\prime \prime}\right)$ as follows:

a. The chain graph $G^{\prime}$ consists of $n+s+1$ nodes, namely $v_{0}^{\prime}, \ldots, v_{n+s}^{\prime}$.

Let $e_{i}^{\prime}=\left(v_{i}^{\prime}, v_{i+1}^{\prime}\right), 0 \leq i \leq n+s-1$.

b. For each path $\left\langle v_{i}, v_{j}\right\rangle \in \mathcal{P}^{\prime}$, add a path to $\mathcal{P}^{\prime \prime}$ :

if $i<j$ add path from node $v_{i}^{\prime}$ to node $v_{j}^{\prime}$ to $\mathcal{P}^{\prime \prime}$, otherwise add path form node $v_{i}^{\prime}$ to node $v_{n+j}^{\prime}$ to $\mathcal{P}^{\prime \prime}$.

6. Call Algorithm for MinCollisions-PMC in chains on instance $\left(G^{\prime}, \mathcal{P}^{\prime \prime}, w\right)$. Color each path in $\mathcal{P}^{\prime}$ with the color of the corresponding path in $\mathcal{P}^{\prime \prime}$.

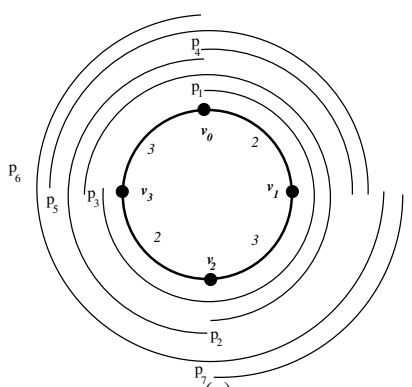

(a)

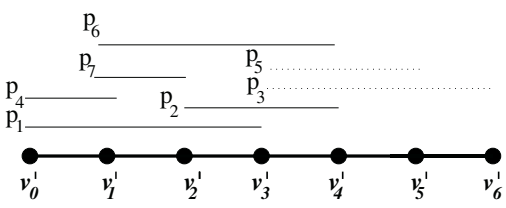

(b)

Fig. 1. An example of how we transform the ring (a) into a chain (b). In (a) we see all the paths in $\mathcal{M}$ and in each edge the available multiplicity. Lets assume that $w=2$. Then $\mathcal{P}_{0}=\left\{p_{3}, p_{5}\right\}$ and we multicolor the remaining paths $p_{1}, p_{2}, p_{4}$

is $S O L \geq|\mathcal{M}|-\left|\mathcal{P}_{0}\right|, \mathcal{P}_{0} \leq w$ and $\mathcal{M} \geq\left(\mu_{\text {min }}-1\right) w\left(\right.$ where $\left.\mu_{\text {min }}=\min _{e \in E} \mu(e)\right)$, we have that the following holds:

$$
\frac{S O L}{O P T} \geq \frac{|\mathcal{M}|-\left|\mathcal{P}_{0}\right|}{|\mathcal{M}|} \geq 1-\frac{\mathcal{P}_{0}}{\mathcal{M}} \geq 1-\frac{w}{\left(\mu_{\text {min }}-1\right) w}=\frac{\mu_{\text {min }}-2}{\mu_{\text {min }}-1}
$$

Thus we have a $\rho=\frac{\mu_{m i n}-1}{\mu_{m i n}-2}=1+\frac{1}{\mu_{m i n}-2}$ Observe that for $\mu_{m i n} \geq 4$, we obtain an approximation ratio $\rho \leq 3 / 2$. 


\section{Iterative Algorithm for Rings and Stars}

Our algorithms use again as a subroutine algorithms for the MAX-MF problem. Initial we have an empty set $\mathcal{P}^{\prime}$. For each color $i(0 \leq i \leq w)$ we find a valid with respect to $\mu$ path packing $\mathcal{P}_{i} \subseteq \mathcal{P}$. Every path in $\mathcal{P}_{i}$ takes color $i$, is removed from $\mathcal{P}$ and is added to $\mathcal{P}^{\prime}$. The proof of Theorem 3 is omitted.

Theorem 3. The iterative algorithm for MAX-PMC is a $1 /\left(1-e^{-1}\right)$ - approximation if an exact algorithm for MAX-MF is used as a subroutine.

When we apply the iterative algorithm for MAX-PMC in rings we get another algorithm.

Corollary 1. The iterative algorithm for MAX-PMC in rings is a 1.58approximation algorithm.

Algorithm 2 for MAX-PMC in rings has worse approximation for instances of the problem where $\mu_{\text {min }}<4$ than the iterative one, but for instances with $\mu_{\text {min }} \geq 4$ the approximation performs better, i.e. $3 / 2 \geq \rho \geq 1$. If $\mu_{\min }$ is very large our algorithm is near optimal, with $\rho$ close to 1 .

Problem MAX-MF can be solved optimally in stars (see [9]), thus we have:

Corollary 2. The iterative algorithm for MAX-PMC in stars is a 1.58approximation algorithm.

\section{Conclusions and Open Problems}

In this paper we present algorithms for MAX-PMC in chains, rings and stars. In rings the solution can be as good as optimal if the minimum fiber multiplicity of the network is large enough, thus improving the proposed algorithm for general networks in 4. All our algorithms are easy to implement, run in polynomial time and have guaranteed worst case behavior. Some open problems for future work include the variation where we we are given requests (pair of nodes) instead of paths. Another interesting version of MAX-PMC is the one, where each request has a profit and the goal is to maximize the total profit of satisfied requests.

\section{References}

1. Nomikos, C., Pagourtzis, A., Zachos, S.: Satisfying a maximum number of prerouted requests in all-optical rings. Computer Networks 42 (2003) 55-63

2. Erlebach, T., Jansen, K.: Maximizing the number of connections in optical tree networks. In: Proceedings of the 9th Annual International Symposium on Algorithms and Computation (ISAAC '98). LNCS 1533 (1998) 179-188

3. Erlebach, T., Pagourtzis, A., Potika, K., Stefanakos, S.: Resource allocation problems in multifiber WDM tree networks. In: Proc. of the 29th Workshop on Graph Theoretic Concepts in Computer Science. LNCS 2880, Springer-Verlag (2003) 218 229 
4. Saad, M., Luo, Z.: On the routing and wavelength assignement in multifiber WDM networks. In: Proceedings of Globecom 2002. (2002)

5. Li, D., Jia, X., Hu, X., Gong, E.Z.: Wavelength assignment for minimizing system blockings in multifiber all-optical WDM networks. Optical Networks 4 (2003) 75-81

6. Carlislie, M., Lloyd, E.: On the k-coloring of intervals. Discrete Applied Mathematics 59 (1995) 225-235

7. Nomikos, C., Pagourtzis, A., Zachos, S.: Routing and path multicoloring. Information Processing Letters 80 (2001) 249-256

8. Adamy, U., Ambuehl, C., Anand, R.S., Erlebach, T.: Call control in rings. In: Proceedings of the 29th International Colloquium on Automata,Languages, and Programming (ICALP 2002). LNCS 2380 (2002) 788-799

9. Garg, N., Vazirani, V.V., Yannakakis, M.: Primal-dual approximation algorithms for integral flow and multicut in trees. Algorithmica 18 (1997) 3-20 